\title{
Inocuidad de los alimentos y riesgo para la salud: el problema del manejo y uso de agroquímicos por pequeños agricultores de la costa central en Perú
}

\section{Food safety and health risk: the problem of agrochemical handling and use by small farmers of central coast in Peru}

\author{
Alfredo Beyer Arteaga ${ }^{1}$, Germán Joyo Coronado ${ }^{1}$, Patricia Rodríguez Quispe ${ }^{1}$, Rubén Darío Collantes \\ González $^{2}$, Fernando Paz Zagaceta ${ }^{1}$ \\ Universidad Nacional Agraria La Molina ${ }^{1}$ \\ Instituto de Investigación Agropecuaria de Panamá ${ }^{2}$ \\ *abeyer@1amolina.edu.pe
}

DOI: https://doi.org/10.26871/killkana_tecnica.v3i2.572

\begin{abstract}
Resumen
El presente estudio abordó la problemática del manejo y uso deficiente de pesticidas mediante la aplicación de un cuestionario a una muestra de pequeños agricultores de la costa central del Perú. Esta problemática es repetitiva y preocupante en diferentes países latinoamericanos, ya que no cuentan con una adecuada regulación de su producción agrícola para el mercado interno. Los resultados indicaron el uso de plaguicidas sin autorización, debido a la falta de productos registrados recomendados, así como el incumplimiento en la dosis recomendada. Adicionalmente, la rotación de ingredientes activos por modo de acción es limitada y no se respetan los términos de carencia, agravándose en el caso de productos destinados al mercado nacional. Así mismo se constató que las recomendaciones de los periodos de reingreso y el uso del equipo de protección personal se incumplen.
\end{abstract}

Palabras clave: Pequeña agricultura, pesticidas, Perú.

\begin{abstract}
The present study addressed the problem of poor pesticide management and use by applying a questionnaire to a sample of small farmers on the central coast of Peru. This problem is repetitive and worrisome in different Latin American countries, since they do not have adequate regulation of their agricultural production for the domestic market. The results indicated the use of pesticides without authorization, due to the lack of recommended registered products, as well as non-compliance with the recommended dose. Additionally, the rotation of active ingredients by mode of action is limited and deficiency terms are not respected, aggravating in the case of products destined for the national market. Likewise, it was found that the recommendations of the re-entry periods and the use of personal protective equipment are not complied with.
\end{abstract}

Keywords: Small agriculture, pesticides, Peru.

\section{INTRODUCCIÓN}

Los grupos de interés relacionados a la producción agrícola, entre agricultores, fabricantes de pesticidas, empresas comerciales, minoristas, autoridades reguladoras, científicos y OrganizacionesNo Gubernamentales (ONGs), por lo general están de acuerdo en que el uso de plaguicidas es necesario, principalmente por la necesidad de alimentar a la creciente población mundial, al mismo tiempo reconocen la naturaleza problemática de la exposición humana a los residuos de plaguicidas[1] .

Se han reportado residuos de plaguicidas por encima de los límites máximos que se permiten (LMRs) en vegetales de alto consumo en China [2], en alimentos procesados a base de cereales para niños e infantes en Ghana [3], en frutas y vegetales provenientes de Asia hacia Europa [4], entre otros. Las evidencias en la India sugieren que los plaguicidas no solo han tenido un efecto negativo en la salud de su población, sino también en el medio ambiente. Se ha detectado la presencia de Compuestos Orgánicos Persistentes (COPs) en muestras de distintos componentes del ambiente, tanto en aire, agua y suelo [5].

También se han identificado herbicidas inhibidores del fotosistema II como el oxadiazon, insecticidas organofosforados como chlorfenvinphos y chlorpyrifos y el orga- 
noclorado endosulfán como los principales plaguicidas, estos tienen efectos tóxicos sobre productores primarios, artrópodos y peces, respectivamente; en mediciones de mezclas de plaguicidas hechas en aguas superficiales de tres importantes cuencas hidrográficas de Portugal [6].

Se detectaron valores de riesgo inaceptables que se relacionaron al cáncer de alachlor y atrazina (atranex, atrazina), así como estimaciones de cocientes de riesgo para los insecticidas chlorpyrifos ethyl (clorpirifos), diazinon (disanon, diazinon) y parathion methyl (herbax) y el herbicida prometryn, por encima de los valores de riesgo aceptables en los principales ríos y lagos del norte de Grecia [7].

Además del impacto en la inocuidad de los alimentos y el medio ambiente, existe un efecto negativo en la salud de los aplicadores que se ven expuestos a estos productos. Agricultores en Tanzania reportaron sentirse enfermos después de aplicaciones rutinarias de plaguicidas, con síntomas que incluyen problemas de la piel y alteraciones del sistema neurológico tales como mareos y dolor de cabeza, así como gastos en salud debido a pesticidas [8]. En el sur de Brasil se encontró correlación entre la exposición a nicotina y plaguicidas, en especifíco organofosforados, y el riesgo de padecer trastornos psiquiátricos menores entre agricultores de tabaco [9]. También se registraron metabolitos de plaguicidas en la orina de pequeños agricultores de la provincia de Chiang Mai, en Tailandia [10].

Estos efectos de residualidad reportados en diferentes partes del mundo, así como el efecto negativo en el medio ambiente y la salud de los aplicadores, guardan una estrecha relación con el manejo y uso que los agricultores dan a los plaguicidas. Así se tiene que productores de hortalizas en Benin hacen uso excesivo de insecticidas y otros plaguicidas al considerar que mayor la cantidad del producto químico está relacionado con la eficacia en el control [11].

Esta situación se repite en Perú, donde no existe una adecuada regulación y monitoreo por parte de las autoridades competentes en cuanto al manejo y uso seguro de plaguicidas entre los pequeños agricultores que abastecen el mercado local. Esto va en detrimento de la inocuidad de los alimentos y la salud de los consumidores nacionales, en contraste con el mayor cuidado e interés de los gobiernos y otras instituciones hacia la agricultura de exportación, tal como sucede en Benín, Etiopía, Ghana y Senegal [12]. En el Perú, existe poca literatura respecto al tema. Pérez et al. (2013)[13] señalaron que se detectó que la presencia de residuos de plaguicidas en hortalizas y frutas es frecuente en México, asimismo se encontró una serie de productos caducos en uso o con almacenamiento inadecuado que son un riesgo para el ambiente.

El presente estudio tiene por objetivo determinar el cumplimiento de las especificaciones técnicas para la aplicación de plaguicidas aprobados en Perú por parte de agricultores de la zona de influencia de la Comisión de Regantes Palo Herbay, en Cañete dedicados al cultivo de fresa. Esto de acuerdo a lo aprobado por el Servicio Nacional de Sanidad
Agraria (SENASA) respecto a la dosis, periodo de carencia y reingreso de los plaguicidas registrados.

\section{MATERIALES Y MÉTODOS}

\section{A. Recolección de información}

La investigación se realizó en el distrito de San Vicente de Cañete, provincia de Cañete, departamento de Lima. La población objetivo fue 59 agricultores productores de fresa durante la campaña del año 2014, pertenecientes a la Comisión de Regantes del Canal Palo Herbay. Para la encuesta se realizó un muestreo simple al azar mediante el método de asignación proporcional [14], utilizado en los proyectos INCAGRO (Innovación y Competitividad para el Agro Peruano, Programa de Inversión Pública del Ministerio de Agricultura del Perú) y otros, siendo factible para poblaciones involucradas mayores a 50 productores de acuerdo a los Estudios de Línea de Base y Salida de Sub-proyectos cofinanciados del referido Proyecto de Investigación y Extensión Agrícola-PIEA- INCAGRO [15], como se aprecia en la Ecuación 1.

$$
n=\frac{\frac{4 P Q}{d^{2}}}{\frac{\frac{4 P Q}{d^{2}}-1}{N}+1}
$$

Donde:

n: tamaño de muestra

$\mathbf{N}$ : población objetivo (Universo)

P: probabilidad de acierto 0.5

Q: probabilidad de error 0.5

d: Error $(10 \%)$

La encuesta es un instrumento de investigación de enorme importancia [16], y es probablemente la mejor aplicación de la teoría y la práctica del muestreo, donde se estudian de forma directa las características de las poblaciones [17].

De igual modo se realizaron entrevistas a diez agricultores sobre el cumplimiento de las recomendaciones del uso de plaguicidas en su cultivo, para obtener información cualitativa con el fin de enriquecer las discusiones. Kvale (2011)[18] indicó que las entrevistas cualitativas han sido un método clave para producir conocimiento científico y profesional, destacándose en la antropología, sociología, ciencias de la educación y de la salud, psicología, entre otros.

\section{B. Variables y procesamiento de datos}

Las variables de estudio estuvieron relacionadas a los factores de aplicación: la utilización de plaguicidas recomendados para el cultivo de fresa en el país, las dosis de aplicación, la rotación de los plaguicidas según modo de acción, el equipo de aplicación que se utilizó, el periodo de carencia o días transcurridos de la última aplicación a la cosecha, el periodo de reingreso a un campo fumigado y el uso de equipos de protección personal (EPP). Los datos obtenidos en campo se contrastaron con las fichas de 
registro de plaguicidas oficiales en el cultivo de la fresa, otorgadas por SENASA, autoridad peruana competente en el tema, previa solicitud por escrito.

Las plagas consideradas para el estudio se determinaron en base al conocimiento de los agricultores para la identificación de las mismas y la importancia del daño económico ocasionado. Estas fueron: gusano de tierra (Agrotis sp.), chinche de la fresa (Nysius sp.), arañita roja (Tetranychus sp.), podredumbre gris (Botrytis cinerea) y oidium de fresa (Sphaerotheca macularis).

La información recolectada en campo con la encuesta, fue tabulada en bases de datos de Microsoft Excel y parte de su procesamiento se realizó mediante tablas y fórmulas en el mismo programa. Luego la información fue codificada y sistematizada para su procesamiento con el programa estadístico SPSS a través de estadísticos descriptivos, tablas de frecuencias, y gráficos de reporte.

\section{RESULTADOS Y DISCUSIONES}

\section{A. Productos comerciales registrados}

Los agricultores de fresa suelen usar plaguicidas no registrados, ya que para este cultivo son pocos los productos registrados por las empresas debido a su limitada área de siembra en el país. Ellos deciden adquirir los productos químicos principalmente por la recomendación de los vendedores de las tiendas de agroquímicos, y en menor medida, por su experiencia en campañas pasadas o recomendación de otros agricultores que usaron el producto. Sobre el particular, FAO (2014)[19] señala que la industria de plaguicidas debe probar mediante procedimientos y métodos de ensayo reconocidos, las propiedades, eficacia, comportamiento y riesgo de cada producto plaguicida en relación con las distintas utilizaciones y condiciones previstas en los países que se utilice. Del mismo modo, presentar tales ensayos para su evaluación por las autoridades gubernamentales competentes de todos los países donde el plaguicida va a ofrecerse para su venta o utilización. IICA (2016)[20] también indica que el producto debe estar registrado en el organismo competente de cada país y autorizado su uso para el cultivo a tratar, lo cual no se viene cumpliendo en el cultivo de fresa, en comparación con las fichas de registro facilitadas por SENASA, el organismo competente en el Perú. En el caso del distrito de Huaral, Yengle et al. (2008)[21] incluso reportaron la utilización de insecticidas de uso restringido y prohibido en el Perú.

Para el control de Agrotis sp. los agricultores usan Lorsban (clorpirifos), Tifon (clorpirifos), Decis (deltametrina) y Cipermex (alfacipermetrina) en un 56,76\%, 18,92\%, $16,22 \%$ y $8,11 \%$ como productos comerciales más importantes respectivamente, sin contar ningún producto para Agrotis en fresa con registro en SENASA.

En el caso de Nysius sp. solo se tiene registro el producto comercial Diamond (diazinon).

Para Tetranychus sp., el producto comercial con mayor porcentaje de uso fue Abamex (abamectina), que no cuenta con registro en fresa y fue usado por el 51,35\% de agricultores. Le siguen en orden de importancia los productos registrados Vertimec (abamectina) con 18,92\% y Oberon (spiromesifen) con 16,22\%.

Para Botrytis cinerea el producto comercial más utilizado fue el producto registrado Rovral (iprodione) con $32,43 \%$, seguido del producto no registrado Cercobin (tiofanate metil) con $21,62 \%$, y Folicur (tebuconazole) con $18,92 \%$ de uso y que cuenta con registro en fresa.

En el caso de Sphaerotheca macularis el producto comercial con registro en fresa Topas (penconazole), fue el más ampliamente difundido con un 72,97\% de uso, seguido por los productos no registrados Rubigan (fenarimol) y Prosper (spiroxamina) con $18,92 \%$ y $16,22 \%$ respectivamente.

\section{B. Dosis}

Las dosis utilizadas para los productos comerciales registrados Bamectin y Topas, fueron mayoritariamente superiores a las recomendadas en su ficha técnica. Para el caso de los productos comerciales Vertimec, Oberon, Folicur y Forte, se reportaron dosis menores a las recomendadas en etiqueta en la mayoría de observaciones. Cabe señalar que la gran parte de los plaguicidas registrados ante el SENASA presentan una dosis única recomendada, y no un rango como debería recomendarse. Al respecto, FAO (2014)[19] afirma que los gobiernos tienen la completa responsabilidad de regular la disponibilidad, distribución y utilización de plaguicidas en sus países, pero la industria y los comerciantes de plaguicidas deben observar las prácticas siguientes en el manejo de plaguicidas y ser capaces de brindar un apoyo técnico eficaz, sostenido por una gestión cabal del producto hasta el nivel del usuario final. De ese modo contribuyen a asegurar el uso efectivo y reducir al mínimo los riesgos, y ello reviste una especial importancia en los países que todavía no han establecido sistemas reguladores adecuados ni servicios de asesoramiento, como es el caso del Perú. Es así que Montoro et al. (2009)[22] reportaron un uso indiscriminado de plaguicidas en los andes centrales del Perú.

Al igual que en la elección de los productos, los agricultores deciden las dosis de los productos químicos principalmente por la recomendación de los vendedores de las tiendas de agroquímicos y por su experiencia en campañas pasadas o recomendación de otros agricultores que usaron el producto.

\section{Rotación por modo de acción}

Los porcentajes de rotación según modo de acción de los plaguicidas de acuerdo a los códigos IRAC (Insecticide Resistance Action Committee) y FRAC (Fungicide Resistance Action Committee), de los respectivos comités contra la resistencia a estos productos, pueden apreciarse en la Tabla I. 
TABLA I: Rotación por modo de acción

\begin{tabular}{|c|c|}
\hline Rotación & $\begin{array}{c}\text { Porcentaje } \\
(\mathbf{\%})\end{array}$ \\
\hline Tetranychus sp. & 29,73 \\
\hline Agrotis $s p$. & 27,03 \\
\hline Nysius sp. & 27,03 \\
\hline Botrytis cinerea & 27,03 \\
\hline Sphaerotheca macularis. & 21,62 \\
\hline
\end{tabular}

Fuente: Elaboración propia

El bajo nivel de rotación de insecticidas se debe a que los agricultores utilizan un solo producto hasta notar que el control ya no es eficiente, y al uso de más de un insecticida con el mismo modo de acción. En este tema, Cisneros (2012)[23] indicó que debe evitarse repeticiones del mismo producto insecticida, no efectuando más de dos aplicaciones del mismo producto por campaña agrícola o por año. Después de la segunda aplicación, el producto debe rotarse con un insecticida que pertenezca a un grupo de modo de acción diferente, es decir, con diferente código IRAC. Por su parte Varés (2006)[24], señaló que, en un programa de tratamientos, deben rotarse los productos fungicidas de modo de acción específica, con otros productos de distinto modo de acción. Cabe destacar que FAO (2014)[19] incide en la importancia de que los gobiernos, la industria de plaguicidas y las organizaciones nacionales e internacionales deben colaborar en el desarrollo y promoción de estrategias para prevenir y controlar la resistencia de las plagas a los plaguicidas, con el fin de prolongar la vida útil de los plaguicidas beneficiosos y reducir los efectos adversos de la resistencia a los plaguicidas.

\section{Equipo de aplicación}

Los distintos equipos de aplicación utilizados por los agricultores pueden apreciarse a continuación en la Tabla II.

TABLA II: Equipo de aplicación

\begin{tabular}{|c|c|c|c|c|}
\hline Equipo de aplicación & $\begin{array}{c}\text { Mochila a palanca } \\
(\mathbf{\%})\end{array}$ & $\begin{array}{c}\text { Motopulverizadora } \\
(\mathbf{\%})\end{array}$ & Ambos ( \%) & Total ( \%) \\
\hline Tetranychus $s p$. & 18,9 & 40,5 & 40,5 & 100 \\
\hline Agrotis $s p$. & 73,0 & 10,8 & 16,2 & 100 \\
\hline Nysius $s p$. & 32,4 & 62,2 & 5,4 & 100 \\
\hline Botrytis cinerea & 40,5 & 40,5 & 18,9 & 100 \\
\hline Sphaerotheca macularis. & 24,3 & 59,5 & 16,2 & 100 \\
\hline
\end{tabular}

Fuente: Elaboración propia

Se reportó un $73 \%$ de utilización de pulverizadora manual de mochila a palanca en el caso de Agrotis sp. Para el resto de plagas y enfermedades el uso exclusivo de motopulverizadora o combinado con mochila a palanca fue mayoritario.

Cisneros (2012)[23] indica que las pulverizadoras manuales son de rendimiento limitado y que se utilizan para cultivos bajos y en propiedades pequeñas y medianas, tal como es el caso del cultivo de fresa y de los pequeños agricultores. El uso de pulverizadora manual de mochila a palanca presenta una eficiencia aceptable para Agrotis sp., debido a que la fresa es un cultivo de porte bajo y las larvas se pueden encontrar durante el día en el suelo al pie de las plantas, estas se alimentan activamente de follaje y tallos por la noche, ocasionando daño grave en la corona[25].

Para el caso de Nysius sp., cuyo daño indirecto es la deformación de frutos pequeños, y sus ninfas más desarrolladas y los adultos pueden distribuirse tanto en frutos próximos al suelo como aquellos de la parte superior de la planta[26], sería más recomendable el uso de motopulverizadora. También en el caso de Tetranychus sp., el cual provoca daños en forma de manchas amarillas en el envés de las hojas, Botrytis cinerea que produce ablandamiento de frutos y Sphaerotheca macularis, que afecta hojas, cáliz de las flores y frutos, es más recomendable el uso de motopulverizadora por cubrir mejor el volumen de la planta.

Sobre este tema, FAO (2014)[19] señala que los gobiernos, la industria de los plaguicidas y la industria de equipos de aplicación deben desarrollar y promover el uso de métodos y equipos que reduzcan al mínimo los riesgos para la salud humana, la salud animal o para el medio ambiente, que aumenten al máximo la eficiencia y eficacia en función de los costos, y organicen una capacitación práctica constante en tales actividades. Adicionalmente, la industria de los equipos de aplicación debería proporcionar a los usuarios información sobre el mantenimiento y uso adecuados de esos equipos, lo cual no suele ocurrir por parte de estas empresas en la zona de estudio. IICA (2016)[20] menciona a su vez que el equipo debe estar en buenas condiciones de mantenimiento y previamente calibrado, sin embargo, esto no es frecuente en los campos observados.

\section{E. Tiempo de carencia}

Los tiempos de carencia o número de días desde la última aplicación a la cosecha respetados por los agricultores, figuran tal como se aprecian en la Tabla III. 
TABLA III: Tiempo de carencia

\begin{tabular}{|l|c|c|c|c|c|}
\hline $\begin{array}{c}\text { Tiempo de carencia } \\
\text { (días promedio) }\end{array}$ & Tetranychus sp. & Agrotis sp. & Nysius sp. & B. cinerea & S. macularis \\
\hline Total & 2,22 & 46,22 & 2,19 & 2.00 & 2.11 \\
\hline Mercado nacional & 1,81 & 46,73 & 1,73 & 1,54 & 1.58 \\
\hline Exportación & 3,5 & 52,5 & 3,00 & 3,25 & 3,5 \\
\hline Ambos & 3,00 & 40,71 & 3,43 & 3,00 & 3,29 \\
\hline
\end{tabular}

Fuente: Elaboración propia

Los agricultores trabajan los tiempos de carencia por plaga sin hacer mayor distinción entre los productos, y los resultados arrojan periodos de carencia similares entre $\mathrm{Te}$ tranychus sp., Nysius sp., Botrytis cinerea y Sphaerotheca macularis con 2,22, 2,19, 2,00 y 2,11 días respectivamente, siendo casi el doble de días para exportación con respecto a mercado nacional. El período de carencia de Agrotis sp. difiere de las otras plagas y enfermedades descritas porque es un problema fitosanitario que se presenta principalmente en etapas iniciales del cultivo, y su última aplicación fue reportada con una anticipación mayor a un mes, resultando 46,22 días en promedio. IICA (2016)[20] señala que uno de los aspectos a tener en cuenta para cosechar, es el tiempo de carencia o tiempo de espera, por lo que es importante llevar un registro de las aplicaciones fitosanitarias.

Cabe resaltar que los periodos de carencia registrados por SENASA son mayores que los apreciados (Vertimec (abamectina), 7 días; Oberon (spiromesifen), 3 días; Rovral (iprodione), 5 días; Topas (penconazole), 7 días.) En este tema, Quiroga y Hauwermeiren (1996)[27] manifestaron que los consumidores chilenos se han visto afectados por el incumplimiento de los periodos de carencia por parte de los productores agrícolas en su país.

\section{F. Periodo de reingreso}

El promedio de periodo de reingreso a la parcela después de una aplicación fue de 0,43 días. El 64,9\% de los agricultores considera que no debe esperar para poder ingresar a realizar labores o revisar su campo. Un $27 \%$ respeta un periodo de reingreso de un día, y el $8,1 \%$ un periodo de reingreso de 2 días. Sobre el particular, IICA (2016)[20] indica que, una vez finalizada la aplicación, el operario deberá señalizar la zona e indicar el tiempo que se deberá esperar para poder reingresar a la misma. Los agricultores desconocen los periodos de reingreso porque no leen las indicaciones que están en las etiquetas de los productos. A esto se suma la urgencia por continuar con las labores de manejo y la ausencia de síntomas relacionados con el ingreso al campo antes de lo recomendado.

\section{G. Equipo de protección personal}

El $51,35 \%$ de los agricultores no utiliza Equipo de Protección Personal (EPP) y un 48,65 \% de los agricultores utiliza Equipo de Protección Personal, pero incompleto, dícese de guantes, botas, lentes o máscaras y mandiles. En otras partes de Latinoamérica, la situación es similar. Escobar et al. (2011)[28] reportaron la falta de uso de protección personal al aplicar plaguicidas del 99,5\% de sus entrevistados, en el distrito de riego número 101 Cuxtepeques en Chiapas, México. FAO (2014)[19] e IICA (2016)[20] remarcan la importancia del uso adecuado del Equipo de Protección Personal completo por parte de los aplicadores.

Sin embargo, los agricultores de fresa de la Comisión de Regantes de Palo Herbay aún no reconocen la importancia de un buen manejo y uso seguro de plaguicidas al no ver efectos y síntomas directos sobre su salud. Escobar et al. (2011)[28] lo reafirman al señalar que el $96 \%$ de los trabajadores agrícolas de la localidad Nueva Libertad, en Concordia, Chiapas; conocen los problemas de salud asociados al manejo inadecuado de plaguicidas y, no obstante, fueron minimizados los riesgos y el peligro.

En el Perú, Montoro et al. (2009)[22] encontraron que los agricultores no cuentan con ropa de protección, y manipulan directamente los plaguicidas durante su preparación y aplicación en las provincias de Chupaca y Concepción en los andes centrales del Perú, lo que representa un peligro para la salud de ellos. Asimismo, Yengle et al. (2008)[21], reportaron que la práctica inadecuada más frecuente entre agricultores en el distrito de Huaral en Perú, durante la fumigación, fue la protección personal incorrecta. Por su parte, Caldas (2011)[29], indicó que la intoxicación por plaguicidas se ha convertido en un problema de salud pública mayor en algunos países en vías de desarrollo debido a la ingestión accidental o intencional y el uso no seguro durante la aplicación de plaguicidas.

\section{CONClusiones}

El nivel de rotación según modo de acción entre los productores es relativamente bajo, y generalmente no se respetan los criterios técnicos de término de carencia, periodo de reingreso y uso de equipo de protección personal durante las aplicaciones.

Los agricultores utilizan de manera común productos comerciales no registrados para fresa y no se ciñen a las dosis recomendadas.

\section{RECOMENDACIONES}

Se recomienda una regulación efectiva de la aplicación de plaguicidas en productos agrícolas destinados al mercado nacional por parte de la autoridad nacional competente, SENASA. 


\section{REFERENCIAS}

[1] W. Verbeke, E. J. Van Loo, F. Vanhonacker, I. Delcour, P. Spanoghe, and J. D. van Klaveren, "Stakeholder attitudes towards cumulative and aggregate exposure assessment of pesticides," Food and Chemical Toxicology, vol. 79, pp. 70-79, 2015.

[2] G. Qin, Y. Li, Y. Chen, Q. Sun, B. Zuo, F. He, N. Shen, G. Jia, and G. Ding, "Pesticide residues determination in China vegetables in 2010-2013 applying gas chromatography with mass spectrometry," Food Research International, vol. 72, pp. 161-167, 2015.

[3] O. Akoto, J. Oppong-Otoo, and P. Osei-Fosu, "Carcinogenic and non-carcinogenic risk of organochlorine pesticide residues in processed cereal-based complementary foods for infants and young children in Ghana," Chemosphere, vol. 132, pp. 193-199, 2015.

[4] L. G. Skretteberg, B. Lyrån, B. Holen, A. Jansson, P. Fohgelberg, K. Siivinen, J. H. Andersen, and B. H. Jensen, "Pesticide residues in food of plant origin from Southeast Asia - A Nordic project," Food Control, vol. 51, pp. 225-235, 2015.

[5] I. C. Yadav, N. L. Devi, J. H. Syed, Z. Cheng, J. Li, G. Zhang, and K. C. Jones, "Current status of persistent organic pesticides residues in air, water, and soil, and their possible effect on neighboring countries: A comprehensive review of India," Science of The Total Environment, vol. 511, pp. 123-137, 2015.

[6] E. Silva, M. A. Daam, and M. J. Cerejeira, "Predicting the aquatic risk of realistic pesticide mixtures to species assemblages in Portuguese river basins," Journal of Environmental Sciences, vol. 31, pp. 12-20, 2015.

[7] E. N. Papadakis, Z. Vryzas, A. Kotopoulou, K. Kintzikoglou, K. C. Makris, and E. PapadopoulouMourkidou, "A pesticide monitoring survey in rivers and lakes of northern Greece and its human and ecotoxicological risk assessment," Ecotoxicology and Environmental Safety, vol. 116, pp. 1-9, 2015.

[8] A. V. Ngowi, T. J. Mbise, A. S. Ijani, L. London, and O. C. Ajayi, "Smallholder vegetable farmers in Northern Tanzania: Pesticides use practices, perceptions, cost and health effects," Crop Protection, vol. 26, pp. 1617-1624, 2007.

[9] N. M. Xavier, A. Gastal, R. Dalke, N. Spada, and V. Iribarrem, "Occupational exposure to pesticides, nicotine and minor psychiatric disorders among tobacco farmers in southern Brazil," NeuroToxicology, vol. 45, pp. 347-354, 2014.

[10] P. Panuwet, T. Prapamontol, S. Chantara, P. Thavornyuthikarn, A. Montesano, R. Whitehead Jr., and D. Barr, "Concentrations of urinary pesticide metabolites in small-scale farmers in Chiang Mai Province, Thailand," Science of The Total Environment, vol. 407, pp. 655-668, 2008.

[11] K. Müller, A. Tiktak, T. J. Dijkman, S. Green, and B. Clothier, "Advances in Pesticide Risk Reduction," Encyclopedia of Agriculture and Food Systems, pp. 17-34, 2014.

[12] S. Williamson, A. Ball, and J. Pretty, "Trends in pesticide use and drivers for safer pest management in four African countries," Crop Protection, vol. 27, pp. 1327-1334, 2008.

[13] M. A. Pérez, H. Navarro, and E. Miranda, "Residuos de Plaguicidas en Hortalizas: Problemática y Riesgo en México," Revista Internacional de Contaminación Ambiental, pp. 45-64, 2013.

[14] R. Collantes and A. Rodríguez, "Sustentabilidad de agroecosistemas de palto (Persea americana Mill.) y mandarina (Citrus spp.) en Cañete, Lima-Perú," Tecnología y Desarrollo, vol. 13, no. 1, pp. 27-34, 2015.

[15] INCAGRO Proyecto de Investigación y Extensión Agrícola, Estudios de Líneas de Base y de Salida de Proyectos Cofinanciados. Lima: Ministerio de Agricultura del Perú, 2006.

[16] V. Díaz de Rada Iguzquiza, Tipos de encuestas y diseños de investigación. 2002.

[17] N. J. Salkind and R. L. Escalona, Métodos de Investigación. Pearson Educación, 1999.

[18] S. Kvale, T. del Amo Martín, and C. Blanco Castellano, Las entrevistas en Investigación Cualitativa. Ediciones Morata, 2011.

[19] FAO, Código Internacional de Conducta para la Gestión de Plaguicidas. Roma: FAO Y OMS, 2014.

[20] IICA, Guía de Formación en Buenas Prácticas Agrícolas en Hortalizas. Buenos Aires: Instituto Interamericano de Cooperación para la Agricultura, 2016.

[21] M. Yengle, R. Palhua, P. Lescano, E. Villanueva, E. Chachi, E. Yana, R. Zaravia, J. Ambrosio, J. Clemente, J. Cornejo, and C. Gutiérrez, "Prácticas de Utilización de Plaguicidas en Agricultores en el Distrito de Huaral-Perú," Revista Peruana de Epidemiología, vol. 12, no. 1, pp. 1-6, 2008.

[22] Y. Montoro, R. Moreno, L. Gomero, and M. Reyes, "Características de uso de plaguicidas químicos y riesgos para la salud en agricultores de la sierra central del Perú," Revista Peruana de Medicina Experimental y Salud Pública, pp. 466-472, 2009.

[23] F. Cisneros, Control Químico de las Plagas Agrícolas. Lima: Sociedad Entomológica del Perú, 2012.

[24] L. Varés Megino, "Resistencia de los patógenos a los fungicidas," Agricultura: Revista agropecuaria, pp. 340-342, 2006.

[25] Davis Universidad de California, "Guía para el manejo de las plagas: Fresas," tech. rep., 2005.

[26] L. Gonzales and S. Díaz, "Nysius sp. (HemipteraLygaeidae) en fresa cultivada en el valle huaral ( $\mathrm{Li}^{-}$ ma)," Revista Peruana de Entomología, pp. 19-21, 1994.

[27] R. Quiroga Martínez and S. V. Hauwermeiren, Chile, globalización e insustentabilidad: Una mirada desde la economía ecológica. Santiago, Chile: Instituto de Ecología Política, Programa de Economía Ecológica, 1996. 
[28] D. Escobar Castillejos, A. Caballero Roque, and J. Rendón Von Osten, "Prácticas de Utilización para Plaguicidas en la Localidad Nueva Libertad, La Concordia, Chiapas," Revista Mexicana de Ciencias Agrícolas, no. 1, pp. 19-30, 2011.

[29] E. D. Caldas, "Pesticide Poisoning in Brazil," Encyclopedia of Environmental Health, vol. 4, pp. 419427, 2011.

Recibido: 25 de marzo de 2019

Aceptado: 13 de junio de 2019 
